\title{
Observation of Dipole-Mode Vector Solitons
}

\author{
Wieslaw Krolikowski, ${ }^{1}$ Elena A. Ostrovskaya, ${ }^{2}$ Carsten Weilnau, ${ }^{1,3}$ Matthias Geisser, ${ }^{1}$ Glen McCarthy, \\ Yuri S. Kivshar, ${ }^{2}$ Cornelia Denz, ${ }^{3}$ and Barry Luther-Davies ${ }^{1}$ \\ ${ }^{1}$ Laser Physics Centre, The Australian National University, Canberra ACT 0200, Australia \\ ${ }^{2}$ Optical Sciences Centre, The Australian National University, Canberra ACT 0200, Australia \\ ${ }^{3}$ Institute of Applied Physics, Darmstadt University of Technology, Darmstadt, Germany
}

(Received 17 April 2000)

\begin{abstract}
We report on the first experimental observation of a novel type of optical vector soliton, a dipolemode soliton, recently predicted theoretically. We show that these vector solitons can be generated in a photorefractive medium employing two different processes: a phase imprinting, and a symmetry-breaking instability of a vortex-mode vector soliton. The experimental results display remarkable agreement with the theory, and confirm the robust nature of these radially asymmetric two-component solitary waves.
\end{abstract}

PACS numbers: 42.65.Tg, 05.45.Yv, 42.65.Hw

Optical spatial solitons in $(2+1)$ dimensions are particlelike solitary waves propagating in a nonlinear bulk medium [1]. The exhaustive research of the past decade has shown that these "light particles" can possess topological phase properties analogous to a charge. Moreover, several light beams can combine to produce a vector soliton with a complex internal structure. This process can be thought of as the formation of a "solitonic molecule" from the constituents of different charge.

Recently, the existence of the most robust "solitonic molecule," the dipole-mode vector soliton, has been predicted [2]. This novel optical vector soliton originates from trapping of a dipole-mode beam by a waveguide created by a fundamental soliton in the copropagating, incoherently coupled, beam. While many other topologically complex structures may be created, it is only the dipole mode that is expected to generate a family of dynamically robust vector solitons [2]. The closest counterexample is the vortexmode vector soliton [3] which has a nodeless shape in one component and a ringlike vortex in the other component. This radially symmetric, vector soliton undergoes a nontrivial symmetry-breaking instability $[2,4]$, which transforms it into a more stable object-radially asymmetric dipole-mode vector soliton, even in an isotropic nonlinear medium.

While the existence and robustness of the dipole-mode vector solitons have been established theoretically for a general model of an isotropic medium with saturable nonlinearity [2], the main question still stands: Is the stability of these asymmetric solitons, as opposed to the radially symmetric vortex-mode solitons, a fundamental phenomenon that can be demonstrated experimentally?

In this Letter we answer this question positively. We observe dipole-mode solitons in strontium barium niobate (SBN) photorefractive crystals experimentally, by employing two different techniques. First, we use a specially fabricated phase mask to create a dipolelike structure in one of the copropagating, mutually incoherent, beams. Second, we observe the symmetry breaking of the vortex- mode soliton and the formation of a dipole-mode soliton, as predicted by the theory.

Theoretical results. - We consider two incoherently interacting optical beams propagating in a bulk, isotropic, saturable medium. The model describes $(2+1)$ dimensional screening solitons in photorefractive (PR) materials in the isotropic approximation [5]. It represents a great simplification with respect to a more realistic treatment taking into account the inherent anisotropy of the nonlocal nonlinear response of a PR crystal [6,7]. However, we will show that it does provide correct qualitative predictions of the phenomena observed experimentally. Moreover, due to the generality of the model, one can expect to observe similar effects in other nonlinear isotropic or weakly anisotropic systems.

The normalized equations for the slowly varying beam envelopes, $E_{1}$ and $E_{2}$, can be written as follows $[5,8]$ :

$$
i \frac{\partial E_{1,2}}{\partial z}+\Delta_{\perp} E_{1,2}-\frac{E_{1,2}}{1+\left|E_{1}\right|^{2}+\left|E_{2}\right|^{2}}=0,
$$

where $\Delta_{\perp}$ is the transverse Laplacian and $z$ is the propagation direction. Stationary, $(2+1)$-dimensional solutions of these equations can be found in the form $E_{1}=$ $\sqrt{\beta_{1}} u(x, y) \exp \left(i \beta_{1} z\right), \quad E_{2}=\sqrt{\beta_{1}} w(x, y) \exp \left(i \beta_{2} z\right)$, where $\beta_{1}$ and $\beta_{2}$ are two independent propagation constants. Measuring the coordinates $x$ and $y$ in the units of $\sqrt{\beta_{1}}$, and introducing the soliton parameter $\lambda=\left(1-\beta_{2}\right) /\left(1-\beta_{1}\right)$, we derive the stationary equations for the normalized envelopes $u$ and $w$ :

$$
\begin{gathered}
\Delta_{\perp} u-u+F(I) u=0, \\
\Delta_{\perp} w-\lambda w+F(I) w=0,
\end{gathered}
$$

where $F(I)=I(1+s I)^{-1}, I=u^{2}+w^{2}$, and $s=1-$ $\beta_{1}$ is the saturation parameter. The limit $s \rightarrow 0$ corresponds to the Kerr medium [4].

As has been recently shown in [2], when one of the beams components, say $w$, is weak, a soliton formed by the $u$ component induces a change in the refractive index of the PR material that traps and guides the weaker $w$ 
component. If the waveguide is induced by a fundamental, bell-shaped soliton, its nonlinear guided modes form a set analogous to the Hermite-Gaussian and LaguerreGaussian linear modes supported by a radially symmetric waveguide [9]. At higher intensities of the trapped beam, the two beams form a vector soliton that is self-trapped by a composite refractive index change induced by both beams. Apart from the bell-shaped vector solitons generated by the ground-state mode of the soliton-induced waveguide, one can anticipate various types of higher-order $(2+1)$-dimensional vector solitons. For example, a radially symmetric vortex-mode vector soliton $[3,4]$ is generated by a nodeless beam guiding the component with a nonzero topological charge and the doughnut structure of a Laguerre-Gaussian $\left(\mathrm{LG}_{0}^{1}\right)$ mode. A radially asymmetric dipole-mode vector soliton [2] has one nodeless component, while the other component has a structure of a Hermite-Gaussian $\left(\mathrm{HG}_{01}\right)$ mode.

The existence domain for the radially asymmetric dipole-mode vector solitons of our model (2) was determined in Ref. [2]. A typical example of such a soliton is shown in Fig. 1(a), along with a phase distribution in a dipole-mode constituent. For any given $s$, the solutions are characterized by a certain cutoff value $\lambda$, when the dipolemode component vanishes. Near the cutoff, the vector soliton can be approximately described by the linear waveguiding theory. With increasing $\lambda$, the $w$ component grows and deforms the effective waveguide generated by the $u$ mode, so that the $u$ component elongates and becomes radially asymmetric. The linear and dynamical stability analysis of these solitons has revealed their astounding dynamical stability, with respect to both small- and large-amplitude perturbations [2]. Overall, the analysis performed in [2] suggested that the dipole-mode vortex solitons would lend themselves to experimental
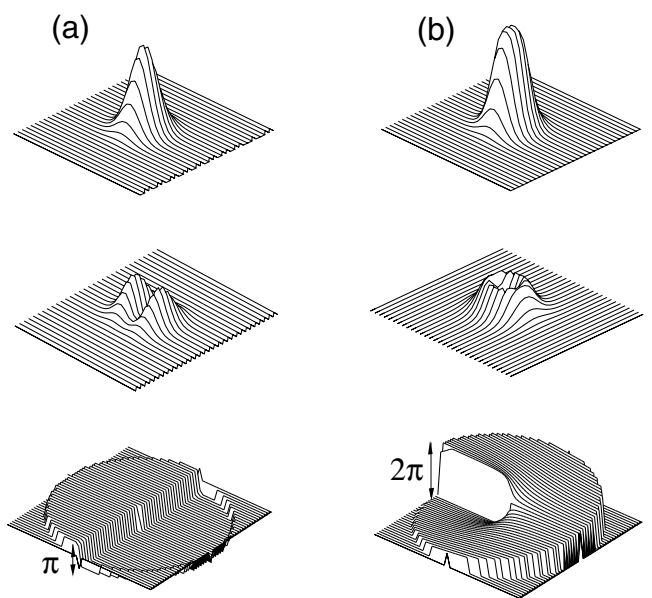

FIG. 1. Examples of constituents of (a) dipole-mode and (b) vortex-mode vector solitons for $s=0.3$ and $s=0.65$, respectively (top and middle row) shown with the phase distributions needed to generate the $w$ component experimentally (bottom row). observation more easily than their unstable vortex-mode counterparts.

The family of radially symmetric vortex-mode solutions of Eqs. (2), in the form: $u=u(r), w=w(r) \exp (i m \phi)$, where $r=\sqrt{\left(x^{2}+y^{2}\right)}$, has been numerically found in $[2,4]$. The $u$ component of this solution has no topological charge and the $w$ component carries a single-charged vortex $(m= \pm 1)$. In Fig. 1(b), we present a typical solution of this family, along with the helical phase distribution needed to generate a vortex in the $w$ component. The dynamical and linear stability analysis of these solutions conducted in $[2,4]$ has proved that all such vortexmode vector solitons are linearly unstable. The instability growth rate is positive for any vanishingly small amplitude of the $w$ component, and it increases rapidly with the vortex intensity.

Remarkably, an unstable vortex-mode soliton displays a symmetry-breaking instability. It always decays into a radially asymmetric dipole-mode soliton with nonzero angular momentum which can survive for very large propagation distances [2]. The breakup of the vortex-mode vector soliton in a saturable medium is a nontrivial effect which, as we show below, can be used to observe dipole-mode vector solitons in PR crystals.

Experimental techniques. - Formation of dipole mode vector solitons has been investigated using an experimental setup shown schematically in Fig. 2. An extraordinary polarized laser beam $(\lambda=532 \mathrm{~nm})$ was split into two parts. The first beam was then transmitted through the phase mask (or glass slide) in order to imprint the required phase structure. In this way, we could obtain either an optical vortex with intensity vanishing in the center of the beam [see Fig. 1(b)] or a dipolelike structure with a phase jump of $\pi$ across the beam along its transverse direction [see Fig. 1(a)] that is perpendicular to the optical axis of the crystal [the axis $(c)$ in Fig. 2]. The second beam was transmitted through the system of spherical or/and cylindrical lenses in order to form either a circular or elliptically shaped spot of desired size. The beams were later combined using the beam splitter BS2 and focused into the input face of the photorefractive crystal. We used two samples of the ferroelectric SBN crystal doped with cerium $(0.002 \%$ by weight $)$. Their dimensions $(a \times$ $b \times c)$ are $(10 \times 6 \times 5) \mathrm{mm}$ or $(15 \times 8 \times 5) \mathrm{mm}$. It is well known that photorefractive crystals biased with strong

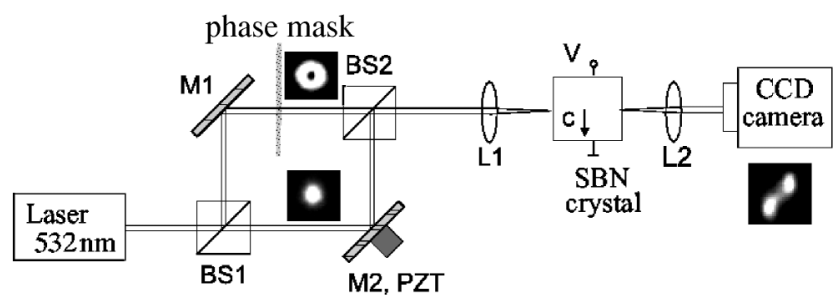

FIG. 2. Experimental setup. 
(a) theory
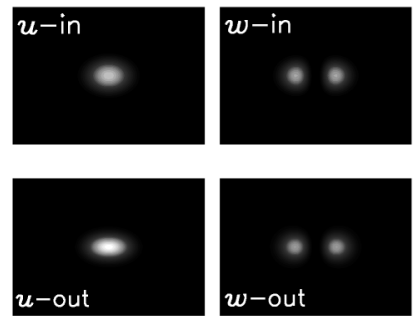

(b) experiment
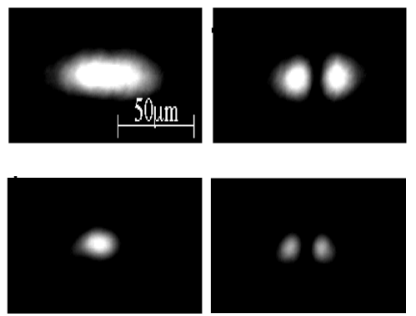

FIG. 3. (a) Results of numerical simulations showing the generation of a stable dipole-mode soliton $(s=0.65$, $\lambda=0.5, z=35$ ). (b) Experimental results showing formation of a dipole-mode soliton (bottom) from the two outof-phase beams (top, right) and copropagating Gaussian beam (top, left). Experimental parameters are $V=2 \mathrm{kV}$, $z=15 \mathrm{~mm}$, and the initial powers of the $u$ and $w$ components $P_{u}=P_{w}=0.6 \mu \mathrm{W}$.

dc electric field exhibit strong positive or negative nonlinearity depending on the polarity of the field [10]. In our case, the SBN crystal was biased with the dc field of $1.5-2.5 \mathrm{kV}$ applied along an optical axis [the axis $(c)$ in Fig. 2]. The resulting photorefractive nonlinearity was of a self-focusing, saturable character.

To control the degree of saturation, we illuminated the crystal with a wide beam derived from a white light source. We estimated the initial (i.e., at the input face of the crystal) degree of saturation to be of the order of unity in all our experiments. Since both the components forming a vector soliton have to be mutually incoherent, one of the constituent beams was reflected from a vibrating mirror mounted on a piezoelectric transducer (M2, PZT). Driving this transducer with an ac signal of $1 \mathrm{kHz}$ imposed a frequency shift onto the beam. This made both beams effectively incoherent inside the photorefractive crystal as the slow photorefractive response does not follow fast changes of the relative phase between the beams. In all our experi- (a) theory
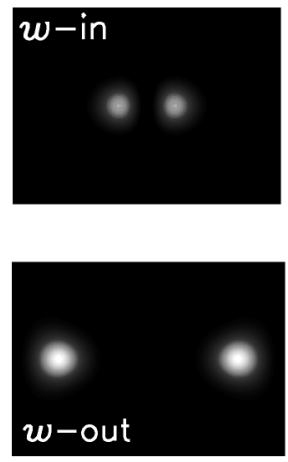

(b) experiment
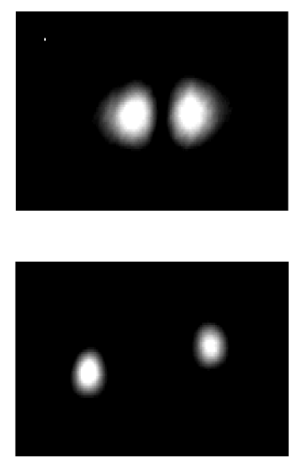

FIG. 4. (a) Results of numerical simulations showing strong repulsion of the input dipole-mode lobes without the second component. (b) Experimental results showing the strong repulsion the two out-of-phase solitons formed by the dipole lobes without the copropagating Gaussian beam [theoretical and experimental parameters as in Figs. 3(a) and 3(b)]. (a)
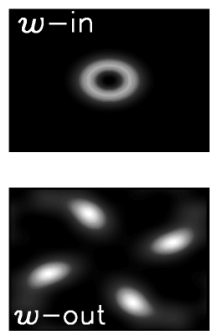
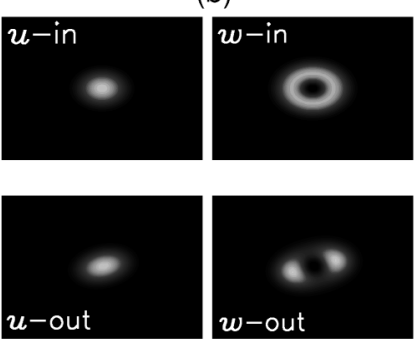

FIG. 5. Suppression of the vortex filamentation due to modulational instability in the presence of a copropagating fundamentalmode beam (see discussion in the text). Numerical results are obtained for $s=0.65$ and $\lambda=0.5$ : (a) $z=35$, (b) $z=90$.

ments the initial power of the beams did not exceed a few microwatts.

Experiment vs theory. - First, we generate the dipolemode soliton by applying a phase mask to one of the input beams to create a dipolelike structure as in Fig. 1(a). If the dipole-mode bearing beam is launched simultaneously with a Gaussian beam shown in Fig. 3(b) (top row), and the two beams are made mutually incoherent, a robust dipole-mode vector soliton is generated [Fig. 3(b), bottom row]. However, when a dipole-mode-bearing beam is launched in the absence of a Gaussian beam, two out-ofphase lobes of the dipole beam form two coherently interacting fundamental solitons that strongly repel each other [Fig. 4(b)].

Our numerical simulations of the original equations (1) provide excellent agreement with the experiment, as is seen in Figs. 3(a) and 4(a). The propagation distance of $z=35$ used in these simulations corresponds to $\approx 9 \mathrm{~mm}$ propagation distance in the PR crystal, which is a priori smaller than the crystal length in most experimental runs.

Next, we examine experimentally a nontrivial breakup of the vortex mode in the presence of the Gaussian beam. Without the Gaussian beam, a scalar vortex exhibits filamentation due to the modulational instability [11,12]. An intermediate stage of a complex vortex filamentation is shown in Fig. 5(a). The corresponding results of an experimental propagation of a single-charge vortex is shown (a)

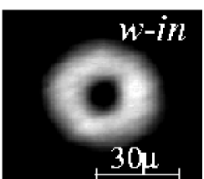

(b)

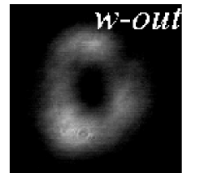

(c)

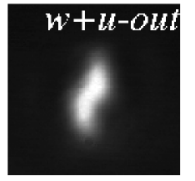

(d)

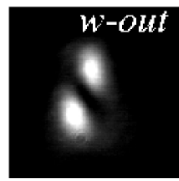

FIG. 6. Experimental demonstration of suppression of the vortex filamentation in the presence of copropagating fundamental-mode beam. Shown are (a) the vortex-bearing input beam, (b) output beam after propagating without the Gaussian beam, (c) output intensity of the dipole-mode soliton formed by two copropagating vortex and Gaussian beams, and (d) intensity of the dipole-mode constituent of the two-component vector soliton. Parameters are $V=2.3 \mathrm{kV}, P_{u}=P_{w}=0.3 \mu \mathrm{W}, z=6 \mathrm{~mm}$. 


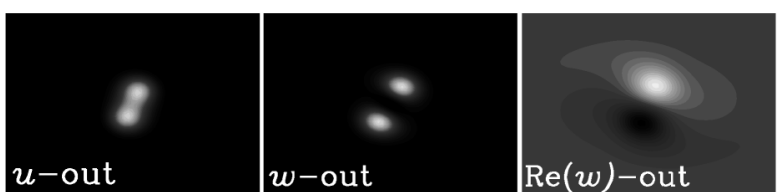

FIG. 7. Numerical results showing the intensity and phase distribution for the dipole-mode component of a vector soliton formed via decay of a vortex-mode vector soliton $(s=0.3$, $\lambda=0.6, z=30)$.

in Figs. 6(a) and 6(b). The filamentation picture is different in the experimental situation owing to the impurities and the inherent anisotropy of the nonlocal nonlinear response of a PR crystal. When the vortex component $w$ is launched simultaneously with the uncharged component $u$, the scenario of the instability development changes dramatically. The vortex-mode instability leads to the formation of a dipole-mode vector soliton, as we demonstrate numerically [see Fig. 5(b)] and experimentally [see Figs. 6(c) and 6(d)]. Importantly, the phase distribution across the profile of the resulting dipole-mode beam calculated numerically and measured in the interference experiment [see Figs. 7 and 8] confirms that the observed localized two-component structure is indeed the dipolemode vector soliton predicted theoretically.
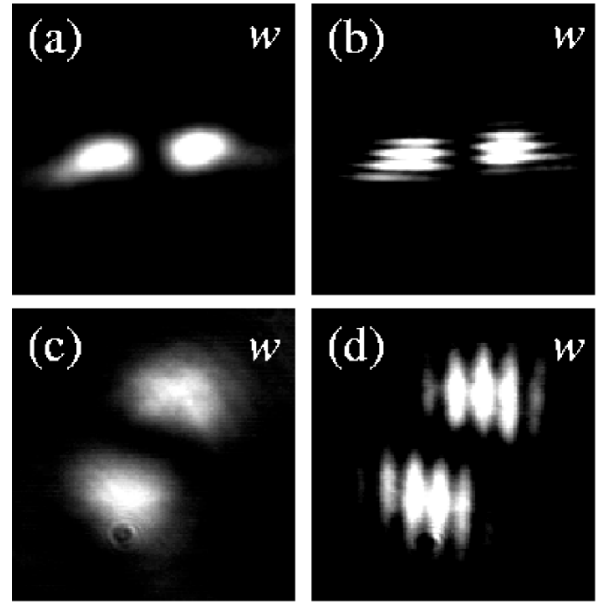

FIG. 8. Experimental results showing $(\mathrm{a}, \mathrm{c})$ the intensities and $(b, d)$ interference pattern for the dipole-mode lobes generated (a,b) via the phase-imprinting experiment, as in Fig. 3(b), and $(\mathrm{c}, \mathrm{d})$ via the symmetry breaking of a vortex-mode soliton, as in Figs. 6(c) and 6(d). The shift seen in the interference fringes of the dipole-mode lobes overlapped with a mutually coherent plane wave $(b, d)$ is the result of the $\pi$ phase difference between the lobes. Parameters are $(\mathrm{a}, \mathrm{b}) V=1.8 \mathrm{kV}, P_{u}=$ $P_{w}=1 \mu \mathrm{W}, z=15 \mathrm{~mm}$; (c,d) corresponds to $V=1.8 \mathrm{kV}$, $P_{u}=1.7 \mu \mathrm{W}, P_{w}=0.4 \mu \mathrm{W}, z=10 \mathrm{~mm}$.
In conclusion, we have generated experimentally a novel type of vector optical soliton in a bulk medium. This soliton has a radially asymmetric structure and originates from trapping of a dipole mode by the soliton-induced waveguide, being much more robust than the corresponding vortex-mode vector soliton. Our experimental results are verified by a systematic comparison with the theory.

C. Weilnau's stay in Australia is supported by the Deutscher Akademisher Austauschdienst (DAAD). This work was supported by the Performance and Planning Fund of the Australian National University.

[1] G. I. Stegeman and M. Segev, Science 286, 1518 (1999).

[2] J. J. Garcia-Ripoll, M. Perez-Garcia, E. A. Ostrovskaya, and Yu. S. Kivshar, Phys. Rev. Lett. 85, 82 (2000). In the $(1+1)$-dimensional geometry, composite multihump solitons were earlier observed experimentally [M. Mitchell, M. Segev, and D. N. Christodoulides, Phys. Rev. Lett. 80, 4657 (1998)], and were shown to be stable [E. A. Ostrovskaya, Yu. S. Kivshar, D. V. Skryabin, and W. Firth, Phys. Rev. Lett. 83, 296 (1999)].

[3] Such (2+1)-dimensional composite solitons were first suggested in Z.H. Musslimani, M. Segev, D. N. Christodoulides, and M. Soljačić, Phys. Rev. Lett. 84, 1164 (2000).

[4] J. N. Malmberg, A. H. Carlsson, D. Anderson, M. Lisak, E. A. Ostrovskaya, and Yu. S. Kivshar, Opt. Lett. 25, 643 (2000).

[5] D. N. Christodoulides, S.R. Singh, M. I. Carvalho, and M. Segev, Appl. Phys. Lett. 68, 1763 (1996).

[6] A. A. Zozulya and D. Z. Anderson, Phys. Rev. A 51, 1520 (1995).

[7] A. Stepken, M. R. Belić, F. Kaiser, W. Królikowski, and B. Luther-Davies, Phys. Rev. Lett. 82, 540 (1999).

[8] A. V. Buryak, Yu. S. Kivshar, M. Shih, and M. Segev, Phys. Rev. Lett. 82, 81 (1999).

[9] L. Gagnon and C. Paré, J. Opt. Soc. Am. A 8, 601 (1991).

[10] M. D. Iturbe-Castillo, P. A. Marquez-Aguilar, J. J. SanchezMondragon, S. Stepanov, and V. Vysloukh, Appl. Phys. Lett. 64, 408 (1994); M. Segev, G. C. Valley, B. Crosignani, P. DiPorto, and A. Yariv, Phys. Rev. Lett. 73, 3211 (1994); M. Shih, M. Segev, G. C. Valley, G. Salamo, B. Crosignani, and P. DiPorto, Electron. Lett. 31, 826 (1995).

[11] For experimental results, see V. Tikhonenko, J. Christou, and B. Luther-Davies, Phys. Rev. Lett. 76, 2698 (1996); J. Opt. Soc. Am. B 12, 2046 (1995); Z. Chen, M. Shih, M. Segev, D. W. Wilson, R.E. Muller, and P. D. Maker, Opt. Lett. 22, 1751 (1997).

[12] For the theory, see W. J. Firth and D. V. Skryabin, Phys. Rev. Lett. 79, 2450 (1997); D. V. Skryabin and W. J. Firth, Phys. Rev. E 58, 3916 (1998). 